\title{
Fuzzy Based Algorithm for Stage-I of Cascaded Intelligent Relaying
}

\author{
Soumyadeep Samonto ${ }^{1,2 *}$, Samarjit Kar ${ }^{3}$, Sagarika Pal'2, Arif Ahmed Sekh4, Bishal Sarkar ${ }^{5}$ \\ ${ }^{1}$ Electrical Engineering Department, Global Institute of Science and Technology, WB, India \\ ${ }^{2}$ Electrical Engineering Department, National Institute of Technical Teachers' Training and Research, Kolkata, WB, India \\ ${ }^{3}$ Department of Mathematics, National Institute of Technology, Durgapur, WB, India \\ ${ }^{4}$ Department of Physics and Technology, UiTThe Arctic University of Norway, Tromsø, Norway \\ ${ }^{5}$ Electrical Engineering Department, Jiaganj College of Engineering and Technology, WB, India \\ Email: ^ssamonto@gmail.com, samarjit.kar@maths.nitdgp.ac.in, sagarikapal@nitttrkol.ac.in, skarifahmed@gmail.com, \\ sarkarbishal1@gmail.com
}

How to cite this paper: Samonto, S., Kar, S., Pal, S., Sekh, A.A. and Sarkar, B. (2021) Fuzzy Based Algorithm for Stage-I of Cascaded Intelligent Relaying. International Journal of Intelligence Science, 11, 31-43. https://doi.org/10.4236/ijis.2021.111003

Received: November 14, 2020

Accepted: January 1, 2021

Published: January 4, 2021

Copyright $\odot 2021$ by author(s) and Scientific Research Publishing Inc. This work is licensed under the Creative Commons Attribution International License (CC BY 4.0).

http://creativecommons.org/licenses/by/4.0/ (c) (i) Open Access

\begin{abstract}
In the present scenario the protection system has become an important issue in the field of the power system. An Intelligent protection system has been introduced in many sectors like low voltage DC breakers, VCB, SF6 and so on. The said protection schemes have been developed to control the moving contacts using intelligent algorithms for tripping overall load against phase to ground faults occurring within the system. The related works have introduced Trapezoidal and Triangular membership functions as input to the fuzzy inference system. It is also found that the Fuzzy Logic Controller has been designed by taking two inputs as current and voltage. The output membership function has been preferred by implementing Trapezoidal and Gaussian membership functions. In this paper, a new concept based over current protection scheme has been introduced. Intelligent relaying technique has been used to trip a particular load against over current fault by introducing multistage cascaded intelligent relaying. Initially, the proposed method is carried out for Stage-I and reported by incorporating fuzzy algorithm by taking current error and current error rates as input using Gaussian membership function to the black box and fed signal to the breaker as output using trapezoidal and triangular membership function respectively to control the loads connected in the system. The best-fit membership function as input to the fuzzy engine is shown here is Gaussian membership function. The analysis reported here by taking both the fault scenario as phase to phase and phase to ground respectively.
\end{abstract}




\section{Keywords}

Fault, Current Error, Protection, Cascaded, Trip, Fuzzy Logic Controller

\section{Introduction}

Incorporation of intelligent system in protection domain has nowadays become one of the most discussed agenda in the field of electrical engineering. Various works have been explored on power system protection by introducing artificial intelligence [1] [2] into various categories of breaker, like [3] [4] [5] [6]. Fault is an unbalanced state that occurs in any normal system. If impedance of any system falls to very low value, then generally fault occurs. Here impedance is actually dynamic resistance. Therefore, as impedance varies inversely with the current, simultaneously current will increase to a maximum surge or peak value. This is why it is necessary to know current error which means the actual value that overshoots the set current due to the huge spikes obtained as impedance falls.

In this area of research, intelligent breaker for controlling distributed load is designed to ensure both over current protection and low voltage protection as well. Considering both inputs and outputs an algorithm is proposed using trapezoidal membership function but for the single phase supply system [7]. Observation of the fault analysis and protective relay operation, intelligent algorithms like ANN and Fuzzy logics have been followed [8]. Investigation report developed on controlling techniques of moving contact behaviors for vacuum circuit breaker based on fuzzy control. The method has been implemented by introducing adaptive fuzzy controlling algorithm [3] [4]. Taking current and voltage as an input to the fuzzy inference system, an algorithm has been developed for fast intelligent relaying which has been presented earlier [9]. Momesso et al. [10] have distinguished between a fuzzy based system and non fuzzy based system with respect to time and current graded protective relaying operating time. Many different approaches have been followed to develop a new kind of intelligent relaying [5] [6].

Some of the articles like [9], have been presented by taking current and voltage as two inputs to the fuzzy inference system. Apart from the fuzzy algorithm, Digital Signal Processing (DSP) has been followed to ensure the reliable operation of the concerned relay in [5] [6]. One of the articles [8], validated the algorithm using Fourier analysis but by designing the output membership function using Gaussian function and assigning it to the output section of the concerned intelligent black box. Few articles have taken membership function as Gaussian for the input as well as output scenarios. Many of them have presented intelligent relaying by taking triangular membership function as input. This contradictory part of selecting a membership function is very difficult to inculpate in any work related to the intelligent relaying scheme. In the previous work, the major problem is with unnecessary tripping as the overall current or voltage 
might not able to give correct perception whereas error and error rates usually notify each micro ampere measure. All of the discussed articles have developed an algorithm to control a single load connected to feeder or bus. No such algorithm has been introduced to control multiple load connected to any single feeder line.

In this paper, a new fuzzy inference system (FIS) has been developed for Stage I of cascaded intelligent relay by taking current error and error rates as input and Gaussian membership function has been assigned to it. The purpose of the cascaded intelligent relaying is to control multiple load connected to a feeder or bus by tripping individual load based on predetermined priority level set to each concerned load connected to a bus or feeder line. The output membership function designed for stage I by taking both trapezoidal and triangular membership function. This entire design is encapsulated inside an intelligent relay block and fed the same to a three-phase breaker for controlling a three-phase system. A brief analysis of successful tripping of concerned intelligent relaying has been performed by taking an over current system model developed under MATLAB environment and reported. Here, we discussed about the best fit membership function as input to the fuzzy engine to be followed in future especially. This is only applicable in case of over current fault protection relaying mechanism. This work ensures the selection of membership function for stage I operation of cascaded intelligent relaying mechanism. The actual motivation of this work is to develop an algorithm for single feeder multiple load connected systems with a better precision tripping mechanism indeed.

This paper has two main contributions: 1) Proposed an algorithm in which current error and current error rates have been taken into account as input to the fuzzy inference system for Stage I. 2) Proposed an idea of multistage cascaded intelligent relying in brief. The rest of the paper presents the same by framing the sections with the proposed method, results and discussions, and subsections framed with cascaded intelligent relaying, block diagram, fuzzy rulebase system, surface view and membership function as well. Finally, the main conclusion and contributions have been discussed in section 4 .

\section{Proposed Method}

The proposed method consists of the linguistic variable based decision making rules for an over current protection system. We have considered an intelligent protection system based on fuzzy algorithm. Overall, 53 rules have been set and the rules are designed by taking Gaussian membership functions. The entire controller system is designed and encapsulated inside a dedicated subsystem for achieving a single logical output to feed the same to the breaker's external port for tripping the system. The overall work is carried out in the MATLAB environment for validating the developed algorithm of an intelligent relay. The purpose of this work is to analyze the implementation of the concerned, intelligent relay by executing the algorithm developed using the fuzzy controller for an over 
current fault protection scheme. In the next subsections, we have discussed the main components of the method.

\subsection{Cascaded Intelligent Relaying: An Overview}

The process of feeding the output of primary FIS to secondary FIS as input is known as cascaded FIS. In designing any intelligent relaying system using a fuzzy logic controller if the theory of cascaded FIS is implemented, then the system is known as cascaded intelligent relaying. As the system is designed with more than one FIS, then the first FIS is taken into account as Stage I and the rest of the cascaded FIS indicated as Stage II, Stage III up to n number of Stages. Thus this particular design is also termed as Multistage based cascaded intelligent relaying. The entire concept is shown in Figure 1. In Figure 1, input to the Stage I initially undergo a process of fuzzification and then ensure output which again follows a defuzzification method and then enters Stage II and finally gives output to the concerned feeder after another second stage defuzzification process.

\subsection{Simulation Block Diagram of Stage-I Intelligent Relaying}

To analyze the characteristic of the system, a deep simulation has been carried out. Artificial fault has been created to record the tripping mechanism of the intelligent relaying system. This fault is initially fed to the system from which the measurement block as per Figure 2 usually fetch the faulty parameters and feed it to the input section of the intelligent relay before disturbing the process. This intelligent relay controls the breaker operation as an external controller and protects the faulty system. Here the process indicates basically load. The relay takes input in the form of current error and current error rates. For better understanding, by taking an example let us get clear about current error. Suppose initial system is designed with a reference input which is constant. Then the source is feeding normal supply to the load. After a while when the system is

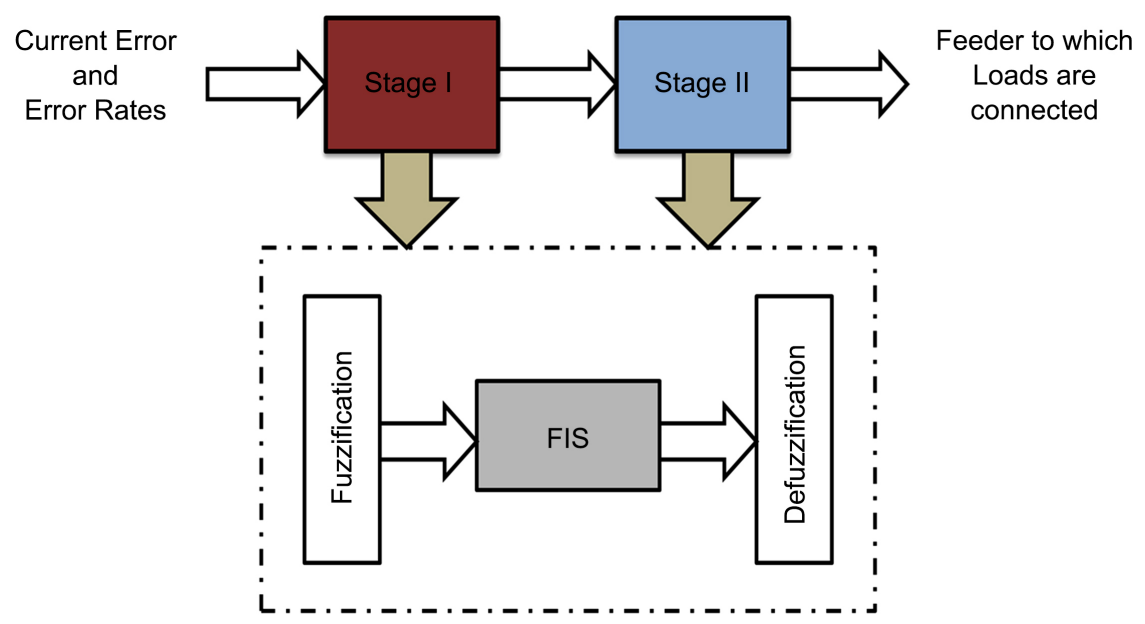

Figure 1. Schematic block diagram of cascaded intelligent relaying showing internal operation of Stage I and Stage II with a dotted border. 


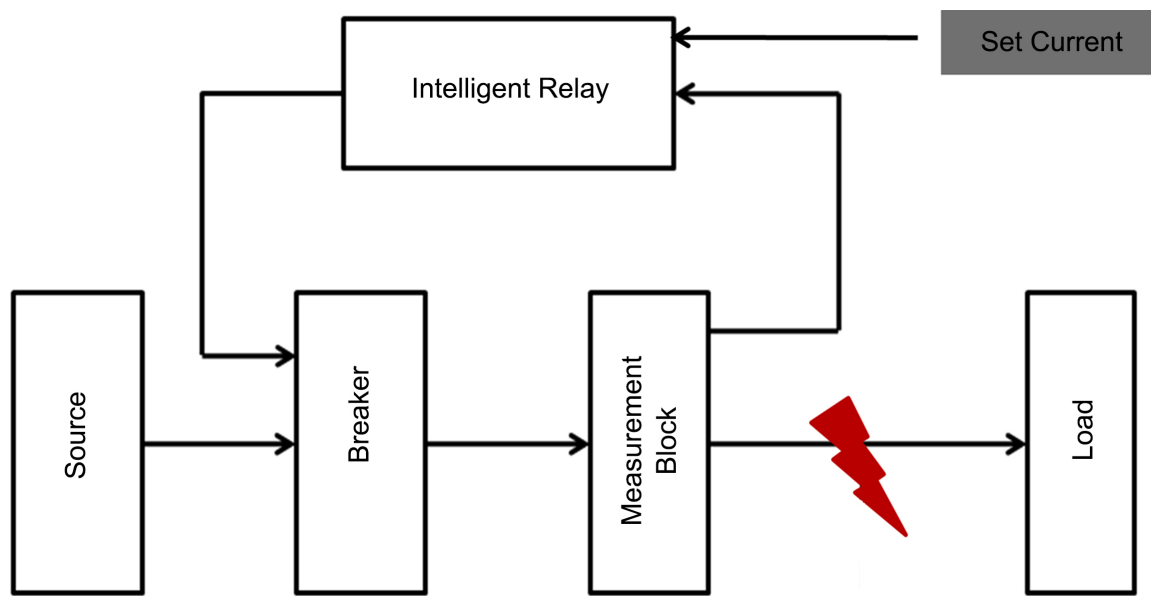

Figure 2. Simulation model block diagram of intelligent relaying.

getting abnormal due to fault then if the fault current exceeds the reference value then errors are introducing to the FIS as first input. If this error is then fed to the same FIS via a differentiator it introduces error rates, which is second input. The control system followed here is feed forward control system. Feed forward system is usually the control system that manipulates the controller output and checks the error before it directly disturbs the process. Figure 2 is the block diagram of the simulation model of the concerned intelligent relaying scheme. The overall setup is designed for three-phase grounded systems. The fault is created and eliminated before it damages the system by fetching the error from the system using FIS incorporated intelligent relay. Relay fed the external signal to the breaker for controlling its contact and tripped the system and protect it from getting damaged.

\subsection{Fuzzy Rulebase System}

The fuzzy algorithm has been incorporated as an intelligent relay to feed the signal to the breaker for tripping the system. Overall, 53 rules are introduced. Among these rules, some rules are taken by accessing current error only, current error rates only and taking both as well. In Table 2, the entire rules have been explained by taking all the 53 rules within a single frame. In this intelligent system among the rules; 5 of them are set by taking only the current errors, 36 of them are set by taking both current error and current error rate and 12 of them are set by taking only current error rate. Since most of the sets are having the same output thus cells are merged to represent all the output under different fault conditions. In case of positive membership function, all of the sets must be reclose. Blank section under current error column indicates that the rules are set by taking only the current error rate and vice versa. Thus, at the side of current error blank section after Z (Zero) cell is the section with only error rates. Likewise the cell beneath PL (Positive Large) of error rates is the sector without error rates but with only errors. Table 1 explains the entire initials of the rules that have been in designing the concerned intelligent relay. 
Table 1. Linguistic variables for Stage-I of cascaded intelligent relaying.

\begin{tabular}{cc}
\hline Membership Function & Indication \\
\hline Zero & $\mathrm{Z}$ \\
Positive Large & $\mathrm{PL}$ \\
Positive Big & PB \\
Positive Medium & PM \\
Positive Small & PS \\
Negative Large & NL \\
Negative Big & NB \\
Negative Medium & NM \\
Negative Small & NS \\
\hline
\end{tabular}

The logic and concept behind designing the rules are not confined to 53 only. Whereas, it is obvious that our main objective is to learn the system with respect to mostly expected unpredictable fault occurs. If the current error is introducing simultaneously error rates have to be there. Thus we consider both error and error rates and designed the rules based on it. Again, if error is present and constant then it is also not necessary to observe any error rates as well. Due to this reason, separate rules are designed by taking only errors and ignoring error rates. On the other side vice versa considered and executed eventually for achieving the better coordination. Though it is very often that the present scenario is of machine learning and still rule based learning we followed just because of the cost effectiveness. Since many under developed countries are not in that situation to afford much so for that reason we have considered an optimal algorithm that could be possible for most of the countries to implement in their system. This in turn actually might help them in overcoming the unnecessary tripping and shutdown scenarios as well.

\subsection{Surface View}

The rules declared for the particular breaker are now thoroughly viewed by justifying the concerned surface view of the FIS developed under MATLAB environment. In Figure 3 an extended figure of the surface view has been explained along with the load axis for the proper validation. The surface view is a GUI tool that lets us examine the output surface of a particular FIS stored in a file, for any one or two inputs.

Here in this case the navy blue coloured trip section is lying beneath -0.6 to 0 along the load axis. And the blue plane is aligned at the zero point. Likewise yellowish plane of Reclose is emerging between the range 0 to 6 along the load axis. Trip section and Reclose sections are a bit different as compared to the no change section. The intermediate section between Reclose and No Change phase is indicated by the Reddish Brown coloured zone as per rules declared and thus 
validating the concerned rules in Table 2 . This change is due to the membership function allotted to these three particular functions. In the next section, a detail discussion on membership function has been presented.

\subsection{Membership Function}

In this section, detailed analysis of the membership functions is carried out. In Figure 4 it is shown how the current error membership function designed to fetch the actual fault scenario of the real world. The Gaussian membership function is taken as the input of the black box because its output is very smooth and outstanding. The mathematical explanation is given in Equation (1) for the betterment of validation indeed.

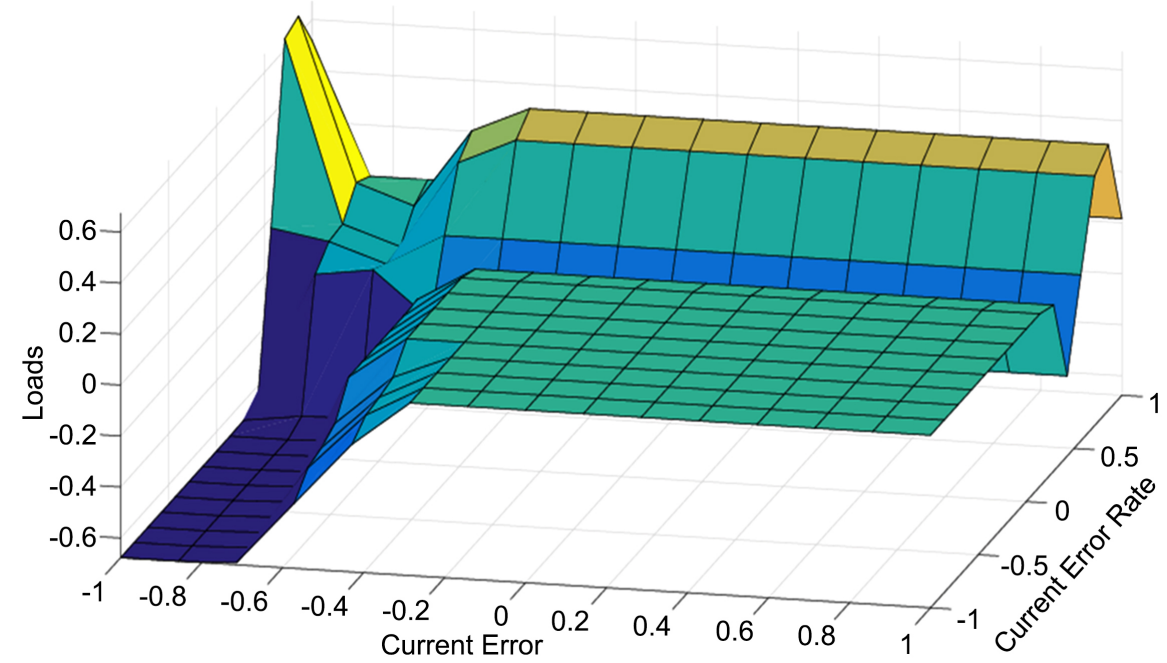

Figure 3. The surface view with the load along $\mathrm{Z}$ axis: Blue colour indicates Trip, Green colour indicates No change, sky blue indicates intermediate between i.e. intersection between no change and trip and Yellow indicates reclose.

Table 2. Rules configuration set for intelligent fault protection scheme.

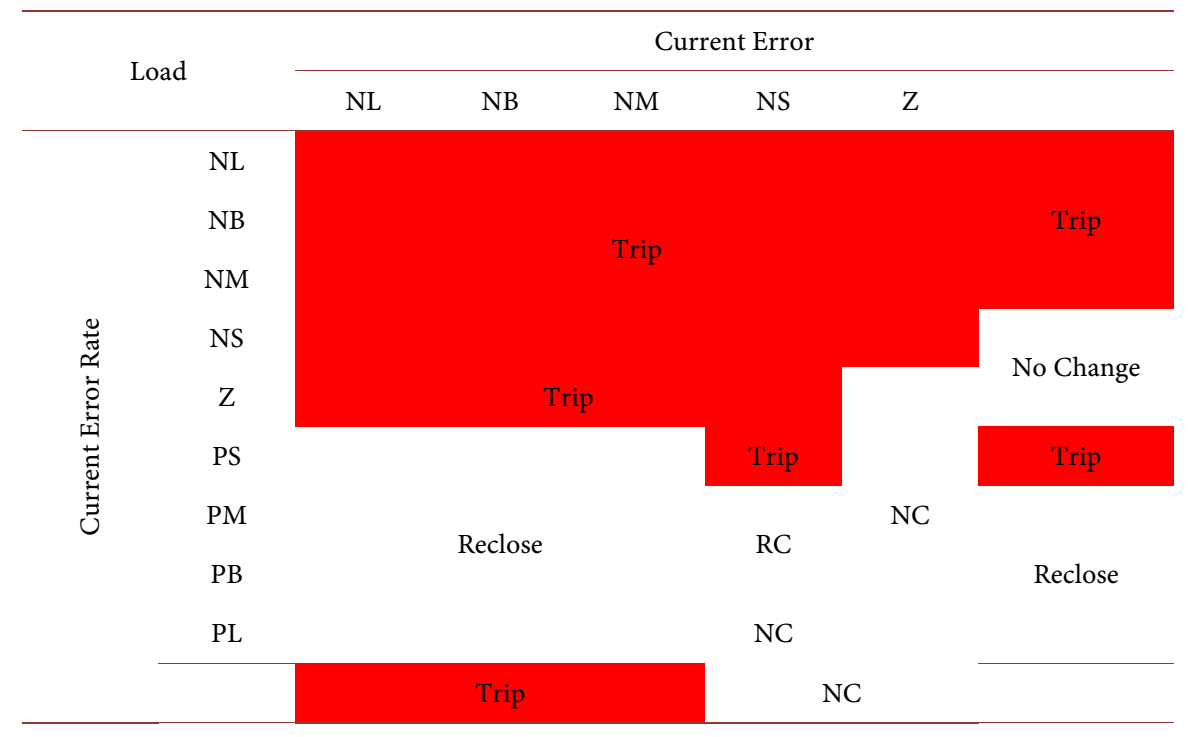




$$
\mu_{A_{i}}(x)=\exp \left(-\frac{\left(c_{i}-x\right)^{2}}{2 \sigma_{i}^{2}}\right)
$$

where $c_{i}$ and $i$ are the center and width of the $i^{\text {th }}$ fuzzy set $A_{i}$, respectively. In this case, it is very essential to implement a Gaussian function during fault analysis as the concerned fault is too dynamic with a huge probability of uncertainty. Since fault will be predicted if the error comes in negative and thus the negative portion of the universe of discourse is basically designed for fault prediction. The positive section is safe and so it is designed with a function named zero ( $\mathrm{Z})$. Similarly, in Figure 5 it is observed that the error and error rates are a bit different as the differentiator of the error is termed as its rates. So here we need both the section and thus designed likewise for obtaining the desired output.

In both the cases, zero is designed with trapezoidal membership function. The universe of discourse is the range of all possible values for an input to a fuzzy system. A fuzzy set is any set that allows its members to have different grades of membership (membership function) in the interval $[0,1]$. The output section of the said protection scheme is given in Figure 6. Here universe of discourse should have to be the same along with error and error rates membership function. Since the negative portion indicates the maximum fault scenario and the positive section is the most safest zone as per rules declared. Thus the negative portion indicates highest priority of error as per input membership function, therefore a trapezoidal membership function has been assigned to the name trip to this section. The Reclose membership function is assigned to the positive section of the discourse of the output membership function. No change condition is

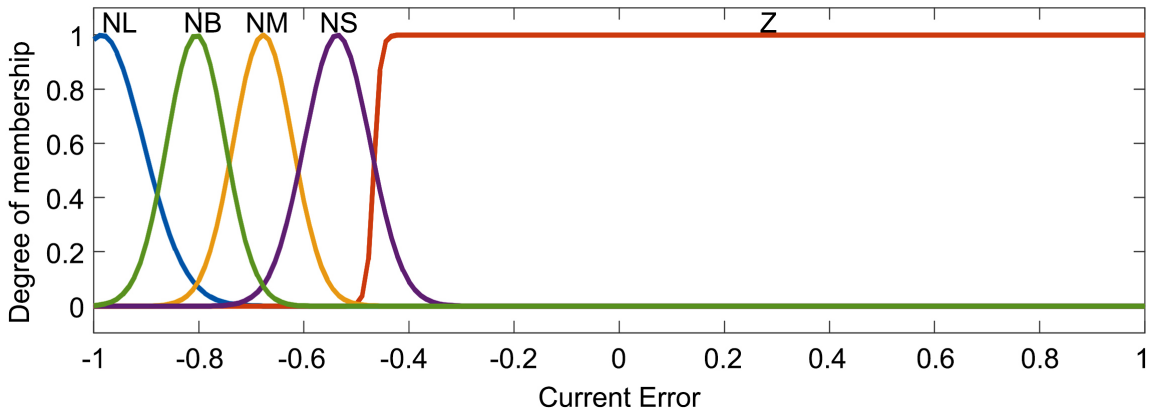

Figure 4. Input membership function of current error.

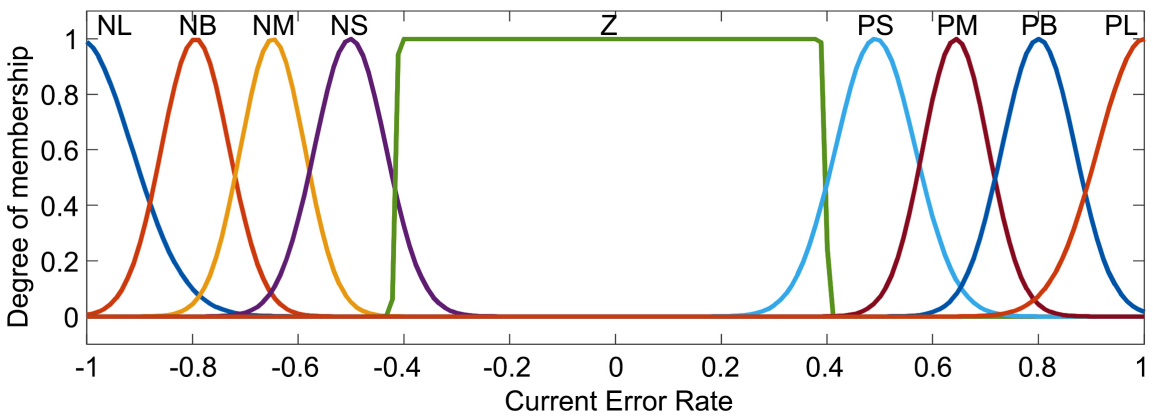

Figure 5. Input membership function of current error rates. 


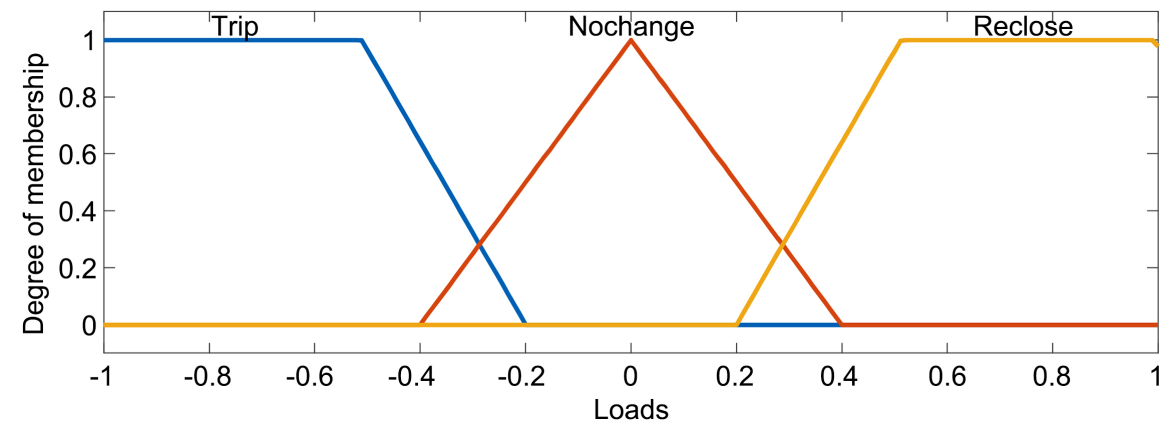

Figure 6. Output membership function of intelligent relay.

assigned to a triangular membership function as in this case center must lie over zero and area coverage needs to lie between the range as given in Figure 6. A mere percentage of its area towards negative section and rest towards positive section as in the rules section Negative Small (NS) error are also allowing the breaker to continue the system with No Change decision. Likewise, positive errors up to a certain range are also following the same decision as well. Finally, before proceeding to the results and discussion section it is better to get clear why it has been followed with a Gaussian function for designing the input variables of the concerned FIS. Apart from this what are the reason behind choosing Trapezoidal and Triangular memberships as the output function of the said black box.

Firstly, as it is clear that the function of the current is time variant and after reaching up to a certain peak it descends mathematically and in the real world as well. Faults usually occur with complex waveforms and it is very easy to fetch the same due to its desired curve obtained as per mathematical equation. On the other side since the Trip and Reclose function of the output section needs a stable and sustained function. As the system trips or recloses in both the cases stay for a long time likewise for this reason only Trapezoidal function has been taken into account as the best suitable function. For any instant decision we generally follow Triangular function and that is why for any sudden change in the operating status of the intelligent relay we have recommended Nochange as another function at the output side for better improvement of the system.

\section{Results and Discussions}

In this section, the detailed analysis of the simulation results is presented. In Figure 7(a) it is clearly explained that the fault is created between Phase $\mathrm{Y}$ and Ground. The distinguished facts are as follows. The $0.1 \mathrm{Sec}$ is the pre-defined step time of the fault creator within the simulation setup.

\subsection{Phase to Ground Fault Analysis}

In Figure 7(a) it is clearly visible that if the fault occurs in case of three phases to ground, then the tripping mechanism is so smooth that just after completion of step time set as per concerned model. 

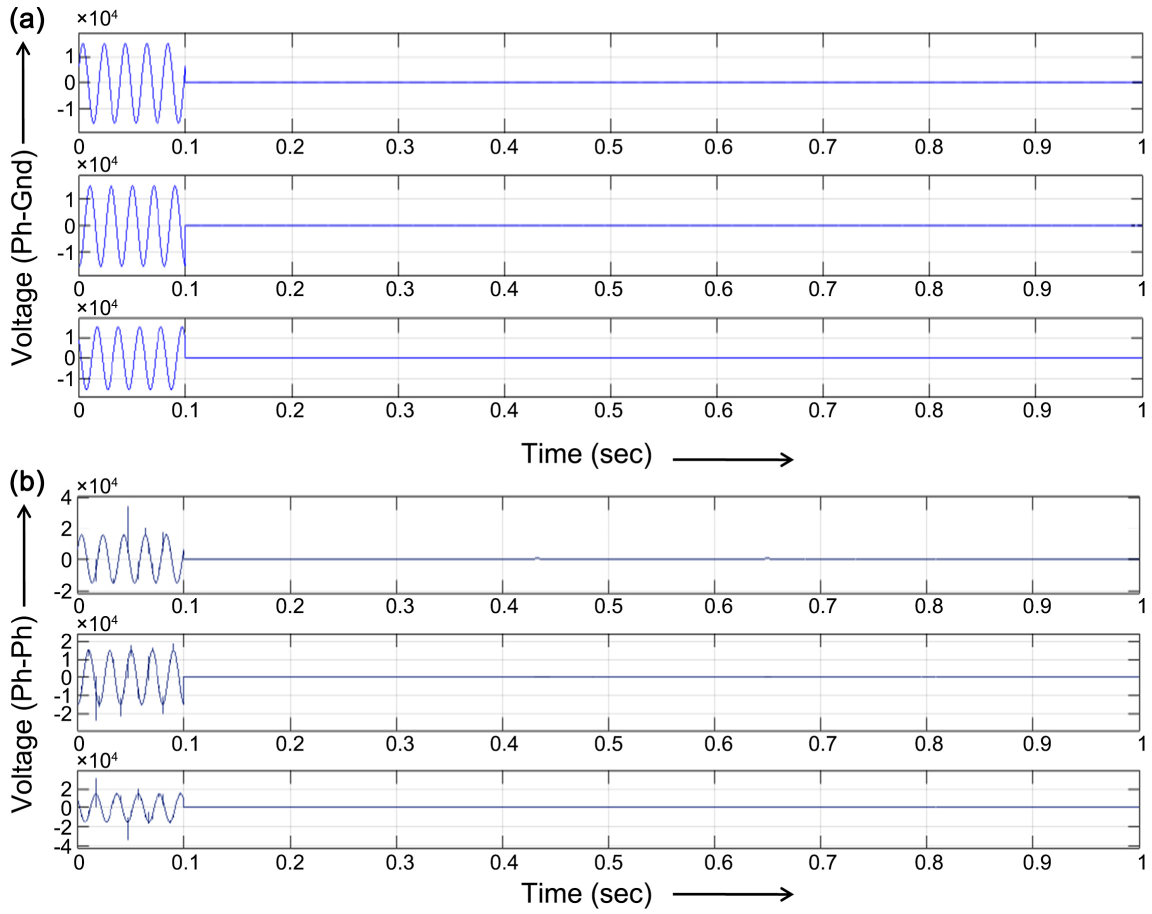

Figure 7. Gaussian Function - Scope for voltage (a) three phase to ground fault (b) phase to phase fault and the step time for fault occurring in the system is set to $0.1 \mathrm{sec}$.

\subsection{Phase to Phase Fault Analysis}

Figure 7(b) indicates the tripping analysis after $0.1 \mathrm{Sec}$ of a simulation run. In case of normal operation, there is some noise recorded due to the absence of ground in the system for analysis as the ground option is unchecked for analyzing the phase to phase fault operation. Figure 8 is clearly validating the changes in current scopes obtained while performing phase to phase and phase to ground fault analysis. From the figure, it is very clear that the simple sinusoidal nature is obtained up to $0.1 \mathrm{Sec}$ of simulation run time after that a random fault current is flowing throughout the simulation with a peak value of around 1500 ampere. The normal peak value is around 200 ampere. All these scope results are obtained by taking Gaussian membership function as the input membership function for FIS set for this particular intelligent fault protection scheme.

To validate the best fit membership function, apart from Gaussian membership function, we have considered two more functions. The two functions are trapezoidal membership function and triangular membership function are considered as input function of the fuzzy inference system and hereby reported as shown in Figures 9-10 respectively.

In Figure 9, at the very end of simulation time, around $0.9 \mathrm{sec}$ later a typical disturbance found in case of trapezoidal membership function. Though it is not that easy to analyze with naked eyes that is why the separate magnified figure provided. Again, in Figure 10, huge disturbances observed between $0.2 \mathrm{sec}$ and $0.4 \mathrm{sec}$ in case of triangular function membership function as input function to the FIS. 


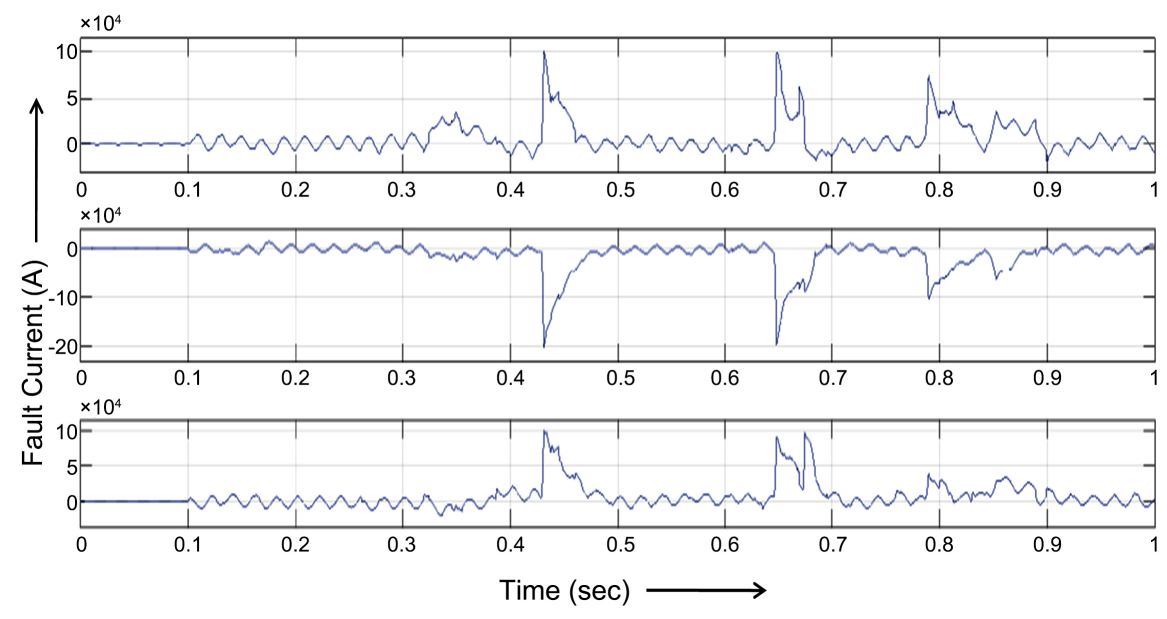

Figure 8. Gaussian Function-Scope analysis of phase to phase fault in case of current ensuring fault within the system.

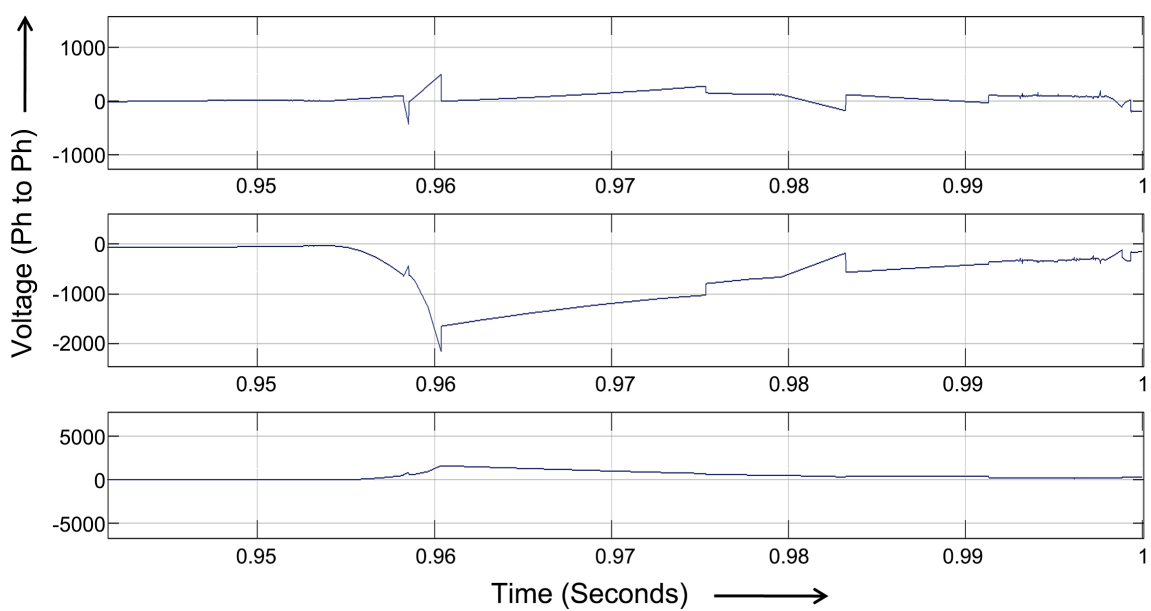

Figure 9. Trapezoidal Function - Scope analysis of phase to phase fault zoom in view of the ranges $0.9 \mathrm{sec}$ to $1 \mathrm{sec}$ and the noise full of output achieved in case of voltage to the load that trips the overall system after $0.1 \mathrm{sec}$ as per model parameter.

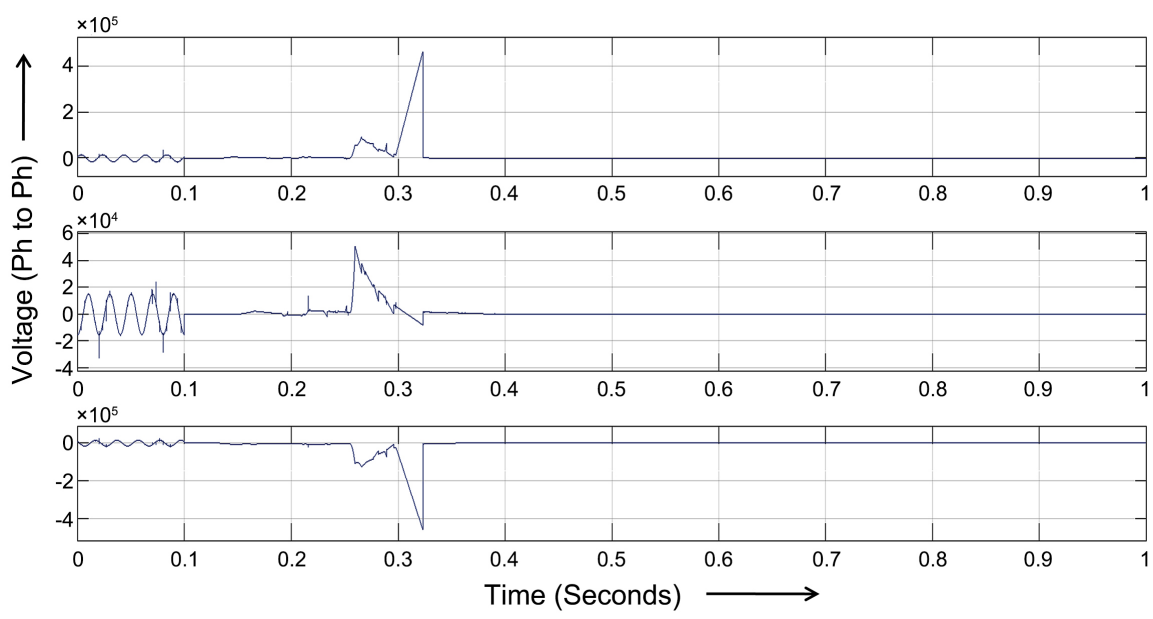

Figure 10. Triangular Function - Scope analysis of phase to phase fault in case of voltage to the load that trips the overall system after $0.1 \mathrm{sec}$ as it ensures fault within the system. 
From this brief comparative study, it is clear that Gaussian function as an input membership function of the fuzzy engine ensures the best output and hence trips the system with a better perception as compared to trapezoidal and triplets as input membership function.

\section{Conclusion}

In this paper, a new fuzzy rule-based intelligent protection system is proposed. We have introduced an intelligent relay to archive the protection scheme. Here intelligent relaying is considered by developing a controller and in there each phase is taken in as an input to an FIS by distinguishing its domain into error and error rates. The system explained here is an over current system. The purpose of selecting Gaussian membership function as an input function is because of its centre which is given in Equation (1). In this work, a comparative analysis is performed among Gaussian, Trapezoidal and Triplets as an input membership function of the fuzzy inference system (FIS) as shown in Figure 7, Figures 9-10 respectively. It is found that if an intelligent relay is to be designed then in case of phase to ground fault Figure 7(a) and phase to phase fault Figure 7(b) condition Gaussian function is the only best suitable input function that must be implemented to design an over current intelligent relay. Thus, from the above explanations, it is very clear that the validation of developing an intelligent relay is confirmed by incorporating Gaussian membership function as an input to the black box for any fuzzy algorithm based relaying system. This intelligent relaying is very much beneficial for introducing the protective zone in the case of smart grid [11] [12] and micro grid as well [13]. The use of intelligent relaying is also necessary and most important for upgrading the protective system configuration of Intelligent Sub Station [14] [15].

\section{Conflicts of Interest}

The authors declare no conflicts of interest regarding the publication of this paper.

\section{References}

[1] Samonto, S., Kar, S., Pal, S. and Sekh, A.A. (2020) Fuzzy Logic Based Multistage Relaying Model for Cascaded Intelligent Fault Protection Scheme. Electric Power Systems Research, 184, Article ID: 106341. https://doi.org/10.1016/j.epsr.2020.106341

[2] Hui, H., Yulong, H., Chao, S., Xi, X., Peng, Y. and Yannan, Z. (2017) Research on the Intelligent Operating Techniques of High Voltage Circuit Breaker. 2017 IEEE Chinese Automation Congress (CAC), Jinan, 20-22 October 2017, 7891-7895. https://doi.org/10.1109/CAC.2017.8244210

[3] Li, T.H., Fang, C.E., Li, W. and Zhou, L.L. (2010) Investigation on Controlling Techniques of Moving Contact Behaviors for Vacuum Circuit Breaker Based on Fuzzy Control. 2010 IEEE Asia-Pacific Power and Energy Engineering Conference, Chengdu, 28-31 March 2010, 1-4. https://doi.org/10.1109/APPEEC.2010.5448251

[4] Liu, Z., Duan, X., Liao, M., Zou, J., Ge, G. and Lv, G. (2016) Investigation on Information Monitoring Technology for Intelligent Vacuum Circuit Breaker. 201627 th International Symposium on Discharges and Electrical Insulation in Vacuum, Vol. 


\section{2, 1-4. https://doi.org/10.1109/DEIV.2016.7763974}

[5] Lee, S.J. and Liu, C.C. (1996) Intelligent Approach to Coordination Identification in Distance Relaying. Proceedings of International Conference on Intelligent System Application to Power Systems, Orlando, 28 January-2 February 1996, 62-67.

[6] Li, K.K., Lai, L.L. and David, A.K. (2000) Stand Alone Intelligent Digital Distance Relay. IEEE Transactions on Power Systems, 15, 137-142. https://doi.org/10.1109/59.852112

[7] Mandal, S.K., Pal, S. and Samonto, S. (2015) Distributed Load Control Using FLC Based MCB. 2015 IEEE International Conference on Electrical, Computer and Communication Technologies (ICECCT), Coimbatore, 5-7 March 2015, 1-7. https://doi.org/10.1109/ICECCT.2015.7225976

[8] Kezunovic, M. (2007) Use of Intelligent Techniques for Analysis of Faults and Protective Relay Operations. 2007 IEEE Power Engineering Society General Meeting, Tampa, 24-28 June 2007, 1-3. https://doi.org/10.1109/PES.2007.386066

[9] Panda, G. and Mishra, R.R. (2000) Fast Intelligent Relaying Using Fuzzy Logic Technique. Proceedings of IEEE International Conference on Industrial Technology, Vol. 2, 159-163.

[10] Momesso, A.E., Bernardes, W.M.S. and Asada, E.N. (2019) Fuzzy Adaptive Setting for Time-Current-Voltage Based Overcurrent Relays in Distribution Systems. International Journal of Electrical Power \& Energy Systems, 108, 135-144. https://doi.org/10.1016/j.ijepes.2018.12.035

[11] Esfahani, M.M. and Mohammed, O. (2020) An Intelligent Protection Scheme to Deal with Extreme Fault Currents in Smart Power Systems. International Journal of Electrical Power \& Energy Systems, 115, Article ID: 105434. https://doi.org/10.1016/j.ijepes.2019.105434

[12] Kawano, F., Baber, G.P., Beaumont, P.G., Fukushima, K., Miyoshi, T., Shono, T., Ookubu, M., Tanaka, T., Abe, K. and Umeda, S. (2010) Intelligent Protection Relay System for Smart Grid. 10th IET International Conference on Developments in Power System Protection (DPSP2010), Manchester, 29 March-1 April 2010, 1-5. https://doi.org/10.1049/cp.2010.0211

[13] Kar, S. and Ranjan Samantaray, S. (2015) A Fuzzy Rule Base Approach for Intelligent Protection of Microgrids. Electric Power Components and Systems, 43, 2082-2093. https://doi.org/10.1080/15325008.2015.1070384

[14] Jinbo, L. and Guoming, Q. (2010) Prospect and Discussion on the Relay System Configuration of Intelligent Substation. IEEE CICED 2010 Proceedings, Nanjing, 13-16 September 2010, 1-5.

[15] El Naily, N., Saad, S.M., Abeid, S. and Saleh, H. (2020) Improved Over-Current Coordination Using Artificial Intelligence in Benghazi MV-Distribution Network Case Study. Proceedings of the 6 th International Conference on Engineering \& MIS 2020, Almaty, Kazakhstan, 14-16 September, 2020, 1-6. https://doi.org/10.1145/3410352.3410809 